\title{
One Electron Changes the Entire Story: NMR versus pNMR
}

\author{
Taye B. Demissie* \\ Centre for Theoretical and Computational Chemistry, \\ Department of Chemistry, UiT The Arctic University of Norway, N-9037 Troms $\phi$
}

\begin{abstract}
B}$ NMR chemical shifts of structurally similar diamagnetic and paramagnetic molecules are obtained using four-component relativistic DFT calculations. The calculated chemical shifts of the diamagnetic molecules are compared with those of the paramagnetic molecules to get an insight on the influence of the unpaired electron on the ${ }^{11} \mathrm{~B}$ chemical shifts.
\end{abstract}

Key words: NMR chemical shifts; Paramagnetic NMR; Boron NMR; density functional theory PACS: 76.60.-k; 33.15.Pw; 31.15.A-; 31.15.aj; 31.15.bw; 31.15.E-; 31.30.jc

\section{INTRODUCTION}

Nuclear Magnetic Resonance (NMR) is a powerful spectroscopic technique that provides detailed information about molecular structures. Its application spans from the vast majority of chemical studies dealing about diamagnetic molecules to a considerable number of studies dealing about paramagnetic molecules, for instance, metalloproteins, optical and magnetic materials as well as related transition metal complexes.

The NMR chemical shifts and spin-spin coupling constants can be accurately determined using both experimental and computational techniques. In the latter case, the isotropic NMR shielding constant of nucleus K, $\sigma_{\mathrm{K}, \text { iso }}$, is expressed as the second derivative of the energy with respect to the nuclear magnetic dipole moment, $\vec{\mu}_{\mathrm{K}}$, and the external magnetic field, $\vec{B}$ :

$$
\sigma_{\mathrm{K}, \text { iso }}=\frac{1}{3} \operatorname{Tr}\left(\frac{\partial^{2} E\left(\vec{\mu}_{\mathrm{K}}, \vec{B}\right)}{\partial \vec{\mu}_{\mathrm{K}} \partial \vec{B}}\right) .
$$

For closed-shell molecules (where the vast majority of studies are dealing about), the absolute shielding constant has two contributions, known as the diamagnetic, $\sigma_{\mathrm{K}}^{\text {dia }}$, and the paramagnetic, $\sigma_{\mathrm{K}}^{\text {para }}$, contributions $[1,2]$ :

$$
\sigma_{\mathrm{K}}=\sigma_{\mathrm{K}}^{\text {dia }}+\sigma_{\mathrm{K}}^{\mathrm{para}} .
$$

Then, the chemical shifts are evaluated as the differences between the absolute shielding constant of some reference compound and the calculated absolute shielding constants of each nucleus of interest in a molecule.

On the other hand, the paramagnetic NMR (pNMR) chemical shift of open-shell molecules has three contributions $[3,4]$ :

$$
\delta_{\mathrm{K}}=\delta_{\mathrm{K}}^{\mathrm{orb}}+\delta_{\mathrm{K}}^{\mathrm{fc}}+\delta_{\mathrm{K}}^{\mathrm{pc}}
$$

where $\delta_{\mathrm{K}}^{\mathrm{orb}}$ is the orbital contribution that results from all paired electrons and $\delta_{\mathrm{K}}^{\mathrm{fc}}$ and $\delta_{\mathrm{K}}^{\mathrm{pc}}$ are Fermi-contact and pseudo-contact terms, respectively, which result from the influence of the unpaired electron(s). The sum of the Fermi- and pseudo-contact terms is commonly known as the paramagnetic contribution, $\delta_{\mathrm{K}}^{\text {para }}$, which can be approximated using Eq. 4 (in terms of $\delta_{\mathrm{K}}^{\mathrm{fc}}$ and $\delta_{\mathrm{K}}^{\mathrm{pc}}$, respectively):

$$
\delta_{\mathrm{K}}^{\text {para }}=\frac{\mu_{e}}{\gamma_{\mathrm{K}}} \frac{S(S+1)}{3 k \mathrm{~T}}\left(g^{\text {iso }} A_{\mathrm{K}}^{\text {iso }}\right)+\frac{\mu_{e}}{\gamma_{\mathrm{K}}} \frac{S(S+1)}{9 k \mathrm{~T}} \operatorname{Tr}\left(g^{\text {ani }} A_{\mathrm{K}}^{\text {dip }}\right)
$$

\footnotetext{
${ }^{*}$ Corresponding author: Dr. Taye B. Demissie; Email: taye.b.demissie@ uit.no
} 
where $\mu_{\mathrm{e}}$ is the Bohr magneton, $\gamma_{\mathrm{K}}$ is the gyromagnetic ratio of nucleus $\mathrm{K}, k \mathrm{~T}$ is the thermal energy, $(2 S+1)$ is the ground state multiplicity, $g^{\text {iso }}$ is the isotropic part of the electron paramagnetic resonance (EPR) $g$-tensor, $A_{\mathrm{K}}^{\text {iso }}$ is the isotropic part of the EPR hyperfine coupling tensor, $g^{\text {ani }}$ is the $g$-tensor anisotropy and $A_{\mathrm{K}}^{\mathrm{dip}}$ is the dipolar part of the EPR hyperfine coupling tensor (details of the formulations can be found in Refs. [5,6] and references therein). The orbital contribution is more often approximated using the chemical shift of structurally identical diamagnetic molecules; whereas the other two are predicted from additional EPR parameters as shown in Eq. 4.

Although the theoretical predictions of NMR and pNMR are different, there are considerable number of papers reporting chemical shifts for paramagnetic molecules obtained using the computational protocols designed for diamagnetic molecules. One best example is the results for a paramagnetic molecule reported by Muhammad et al. [7] obtained using the same methodology designed for diamagnetic molecules. Since the unpaired electron introduces extra contributions, the trend of the chemical shifts could be wrong besides the huge quantitative error introduced. As such, demonstrating the effect of the unpaired electron on the chemical shifts of structurally identical molecules is of interest. Therefore, this contribution compares the NMR chemical shifts of the diamagnetic (closed-shell) molecules nido-9- $\mathrm{CO}-7,8-\mathrm{C}_{2} \mathrm{~B}_{9} \mathrm{H}_{11}(\mathbf{1 a})$ and $\mathrm{Co}(\mathrm{CO})_{2}\left(\eta^{5}-10-\mathrm{CO}-7,8-\mathrm{C}_{2} \mathrm{~B}_{9} \mathrm{H}_{10}\right)(\mathbf{2 a})$ with the corresponding paramagnetic (open-shell) molecule $\left[\mathrm{Co}(\mathrm{CO})_{2}\left(\eta^{5}-10-\mathrm{CO}-7,8-\mathrm{C}_{2} \mathrm{~B}_{9} \mathrm{H}_{10}\right)\right]^{+}(\mathbf{2 b})$. See the spin-density plots and atom numberings in Figure 1.

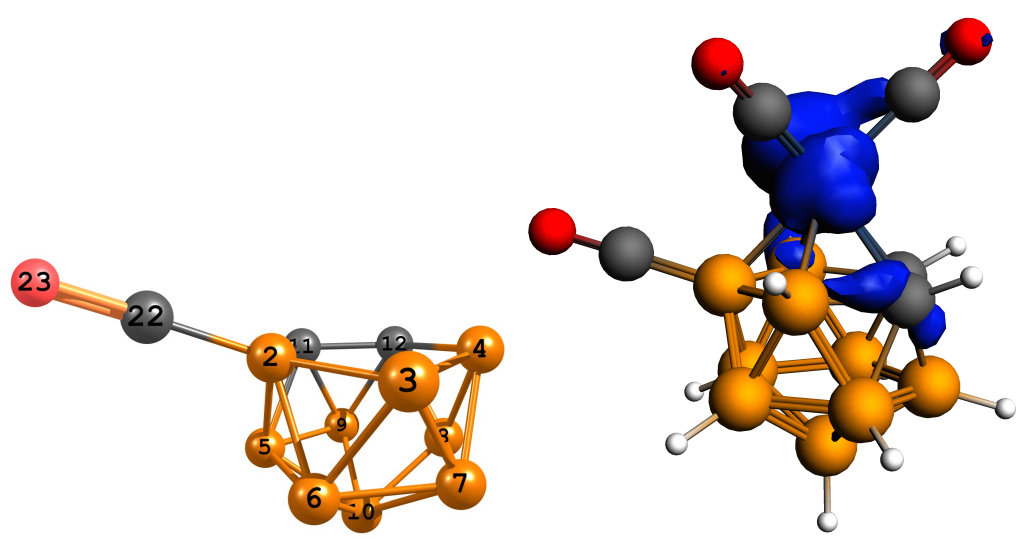

Figure 1: (Left) is the atom numbering followed in this study; hydrogen atoms are removed for clarity. (Right) is the spin-density plot of the paramagnetic molecule $\mathbf{2} \mathbf{b}$.

\section{COMPUTATIONAL DETAILS}

The molecular geometries are optimized using the spin-orbit zeroth-order-regular approximation (SO-ZORA) [8, 9] as implemented in the Amsterdam Density Functional (ADF, version 2014.01) program package [10] using the PBE0 functional [11] and the all-electron triple- $\zeta$ double polarized (TZ2P) Slater-type basis sets [12]. All geometry optimizations were confirmed to be real minimum by performing frequency calculations at the same level of theory. The NMR and pNMR calculations were performed using the four-component matrix DiracKohn-Sham (DKS) relativistic Hamiltonian as implemented in the program package ReSpect [13]. The PBE [14] and PBE0 [11] functionals together with the Dyall's relativistic all-electron valence triple- $\zeta$ (dyall-vtz) basis sets [15] were used in these four-component relativistic calculations. GIAOs were employed for calculation of NMR parameters where the XC kernel was evaluated using SVWN5 potential. Additional test calculations were also performed using XLDA. The SCF calculations were performed with a finite-size nucleus model employing a Gaussian charge distribution, whereas the point model for the magnetic moment distribution was assumed for the EPR and NMR calculations.

\section{RESULTS AND DISCUSSION}

The ${ }^{11} \mathrm{~B}$ NMR chemical shifts of the diamagnetic molecules calculated using the PBE and PBE0 functionals are presented in Table 1 together with the results obtained by modifying the exact-exchange admixture of the PBE0 functional. The results obtained using PBE are in poor agreement with the experimental results. The quality of the 
PBE0 results are improved by manipulating the exact-exchange admixture in the exchange-correlation functional. The standard $25 \%$ exact-exchange is unable to reproduce the experimental results of the ${ }^{11} \mathrm{~B}$ NMR chemical shifts.

Significantly improved results are obtained with a customized PBE0(10\%) functional which includes only $10 \%$ exact-exchange admixture instead of the default $25 \%$ in PBE0. Results obtained from test calculations using PBE0 $(40 \%)$ are also in poor agreement with the experiment. Hence, it appears that PBE0(10\%) is sufficient enough to reproduce the ${ }^{11} \mathrm{~B}$ NMR chemical shifts. This is also further confirmed using test calculations on ${ }^{11} \mathrm{~B}$ NMR chemical shifts of other several molecules.

Table 1: Benchmarking the PBE and PBE0 functionals with varying amount of the exact-exchange admixture (given as $\%$ in parentheses) of the exchange-correlation functional against available experimental ${ }^{11} \mathrm{~B}$ NMR chemical shifts (in ppm) of the diamagnetic molecules. All the calculated results are obtained using the four-component relativistic Hamiltonian and the Dyall-vtz basis sets. ${ }^{a}$

\begin{tabular}{|c|c|c|c|c|c|c|c|c|c|c|}
\hline Boron atom & 2 & 3 & 4 & 5 & 6 & 7 & 8 & 9 & 10 & $\mathrm{MAD}^{b}$ \\
\hline & \multicolumn{10}{|c|}{ nido-9- $\mathrm{CO}-7,8-\mathrm{C}_{2} \mathrm{~B}_{9} \mathrm{H}_{11}, \mathbf{1 a}$} \\
\hline PBE & -42.61 & -22.62 & -18.77 & -21.28 & -4.67 & -30.18 & -0.03 & -26.19 & -36.09 & 5.21 \\
\hline $\operatorname{PBE0}(10 \%)$ & -36.23 & -10.46 & -13.62 & -16.96 & 3.14 & -20.94 & -3.07 & -22.83 & -28.29 & 2.75 \\
\hline $\operatorname{PBE0}(25 \%)$ & -30.04 & 0.02 & -7.95 & -12.84 & 7.45 & -12.34 & -3.39 & -15.26 & -19.09 & 7.65 \\
\hline $\operatorname{PBE} 0(25 \%)^{c}$ & -39.73 & -22.17 & -18.11 & -19.67 & -2.78 & -29.01 & 1.72 & -23.56 & -34.85 & 4.13 \\
\hline \multirow[t]{2}{*}{ Exp. ${ }^{d}$} & -31.5 & -14.2 & -17.3 & -18.4 & -0.1 & -19.5 & -0.1 & -24.1 & -30.5 & - \\
\hline & \multicolumn{10}{|c|}{$\left[\mathrm{Co}(\mathrm{CO})_{2}\left(\eta^{5}-10-\mathrm{CO}-7,8-\mathrm{C}_{2} \mathrm{~B}_{9} \mathrm{H}_{10}\right)\right], \mathbf{2 a}$} \\
\hline PBE & -21.93 & -30.04 & -20.75 & -23.24 & -16.76 & -16.07 & -22.48 & -22.04 & -2.23 & 4.82 \\
\hline PBE0(10\%) & -11.99 & -23.94 & -17.10 & -20.37 & -8.52 & -9.99 & -22.96 & -22.14 & -3.00 & 2.35 \\
\hline $\operatorname{PBE0}(25 \%)$ & -3.07 & -18.85 & -13.80 & -16.71 & -2.88 & -5.86 & -21.27 & -18.35 & -1.94 & 4.28 \\
\hline $\operatorname{PBE0}(25 \%)^{c}$ & -22.33 & -31.29 & -21.04 & -23.50 & -15.08 & -14.49 & -22.66 & -19.88 & -6.61 & 4.55 \\
\hline Exp. ${ }^{d}$ & -15.7 & -23.9 & -15.7 & -17.1 & -11.1 & -11.1 & -18.6 & -18.6 & -4.1 & - \\
\hline
\end{tabular}

$\bar{a}$ The chemical shielding constants for $\mathrm{B}_{2} \mathrm{H}_{6}$ are: $\mathrm{PBE}=75.43, \mathrm{PBE} 0(10 \%)=90.74$, and $\mathrm{PBE} 0(25 \%)=107.70$, whereas its experimental chemical shift referenced to $\mathrm{BF}_{3} \cdot \mathrm{OEt}_{2}$ is $16.6 \mathrm{ppm}$.

${ }^{b} \mathrm{MAD}$ is the mean absolute deviation of the methods relative to the experimental values.

${ }^{c}$ xLDA was used for the MAG calculations.

${ }^{d}$ Taken from Ref. [16].

Now let us turn our attention to Table 2 where the effect of the single electron is presented. We can easily notice that $\sigma^{\text {iso }}$ of $\mathbf{2 a}$ and $\sigma^{\text {orb }}$ of $\mathbf{2 b}$ are in qualitative agreement with each other; which is in line with the Ramsey theory of NMR [2]. Moreover, from the table we also see that the Fermi-contact term is dominant in most cases over the orbital contribution. This causes a large difference between the chemical shifts of the diamagnetic and paramagnetic molecules (see the $\Delta \delta^{\text {iso }}$ values in Table 2), which indicates that approximating the pNMR chemical shifts of paramagnetic molecular systems either by using the methodologies designed for diamagnetic molecules or by approximating using only the orbital contribution leads to wrong conclusions since the effect of the unpaired electron(s) is not taken into consideration.

\section{CONCLUSIONS}

In conclusion, this contribution demonstrated the effect of the unpaired electron on the chemical shifts of diamagnetic and paramagnetic molecules. The appropriate use of the theoretical methodologies designed for the calculation of pNMR chemical shifts (especially for transition metal complexes) is very important for a complete spectral assignments. Although there are no well-established 'black-box' or 'direct' approaches like that of the diamagnetic molecules, the methods used to predict pNMR parameters are nowadays well improved and are being used for related chemical studies. This is very important for the investigation of paramagnetic systems by NMR and to determine their structures by the support of theoretically calculated pNMR parameters and consequently leading to a better understanding of the molecular systems. 
Table 2: Comparison of NMR and pNMR ${ }^{11} \mathrm{~B}$ chemical shifts calculated using DKS/PBE0(10\%)/Dyall-vtz. ${ }^{a}$

\begin{tabular}{|c|c|c|c|c|c|c|c|c|}
\hline \multirow{2}{*}{ Atom } & \multicolumn{2}{|c|}{$2 \mathbf{a}$} & \multicolumn{5}{|c|}{$2 \mathrm{~b}$} & \multirow{2}{*}{$\Delta \delta^{\text {iso }, b}$} \\
\hline & $\sigma^{1 S O}$ & $\delta^{1 \mathrm{SO}}$ & $\sigma^{\text {orb }}$ & $\sigma^{\mathrm{fc}}$ & $\sigma^{\mathrm{pc}}$ & $\sigma^{\mathrm{sum}}$ & $\delta^{1 \mathrm{SO}}$ & \\
\hline 2 & 119.33 & -11.99 & 96.58 & 238.64 & -2.99 & 332.23 & -224.89 & -212.90 \\
\hline 3 & 131.28 & -23.94 & 132.92 & 461.54 & -0.24 & 594.22 & -486.88 & -462.94 \\
\hline 4 & 124.44 & -17.10 & 118.41 & -5.83 & -0.98 & 111.60 & -4.26 & 12.84 \\
\hline 5 & 127.71 & -20.37 & 122.43 & 114.55 & -1.10 & 235.88 & -128.54 & -108.17 \\
\hline 6 & 115.86 & -8.52 & 108.87 & 127.43 & -0.33 & 235.97 & -128.63 & -120.11 \\
\hline 7 & 117.33 & -9.99 & 111.66 & -10.55 & -0.68 & 100.43 & 6.91 & 16.90 \\
\hline 8 & 130.30 & -22.96 & 129.77 & 104.56 & 1.53 & 235.85 & -128.51 & -105.55 \\
\hline 9 & 129.48 & -22.14 & 120.26 & -4.30 & -1.72 & 114.24 & -6.90 & 15.24 \\
\hline 10 & 110.34 & -3.00 & 102.30 & -33.62 & 0.25 & 68.92 & 38.42 & 41.42 \\
\hline
\end{tabular}

\section{ACKNOWLEDGMENTS}

This work has received support from the Research Council of Norway through Grant № . 179568/V30 and has benefited from computer time provided by the Norwegian supercomputing program NOTUR (Grant No. NN4654K).

\section{REFERENCES}

1. N. F. Ramsey, Phys. Rev. 1950, 78, 699.

2. N. F. Ramsey, Phys. Rev. 1951, 83, 540.

3. R. J. Kurland and B. R. McGarvey, J. Magn. Reson. 1970, 2, 286.

4. H. M. McConnell and D. B. Chesnut, J. Chem. Phys. 1958, 28, 107.

5. H. M. McConnell and R. E. Robertson, J. Chem. Phys. 1958, 29, 1361.

6. S. Komorovsky, M. Repisky, K. Ruud, O. L. Malkina and V. G. Malkin, J. Phys. Chem. A 2013, 117, 14209.

7. S. Muhammad, H. Xu, Y. Liao, Y. Kan and Z. Su, J. Am. Chem. Soc. 2009, 131, 11833.

8. E. van Lenthe, E. J. Baerends and J. G. Snijders, J. Chem. Phys. 1994, 101, 9783.

9. E. van Lenthe, A. Ehlers and E. J. Baerends, J. Chem. Phys. 1999, 110, 8943.

10. E. J. Baerends, J. Autschbach, A. Berces, F. M. Bickelhaupt, C. Bo, P. M. Boerrigter, L. Cavallo, D. P. Chong, L. Deng, R. M. Dickson, D. E. Ellis, M. van Faassen, L. Fischer, T. H. Fan, C. Fonseca Guerra, S. J. A. van Gisbergen, J. A. Groeneveld, O. V. Gritsenko, M. Gruning, F. E.Harris, P. van den Hoek, C. R. Jacob, H. Jacobsen, L. Jensen, G. van Kessel, F. Kootstra, E. van Lenthe, D. A. McCormack, A. Michalak, J. Neugebauer, V. P. Osinga, S. Patchkovskii, P. H. T. Philipsen, D. Post, C. C. Pye, W. Ravenek, P. Ros, P. R. T. Schipper, G. Schreckenbach, J. G. Snijders, M. Sola, M. Swart, D. Swerhone, G. teVelde, P. Vernooijs, L. Versluis, L. Visscher, O. Visser, F. Wang, T. A. Wesolowski, E. van Wezenbeek, G. Wiesenekker, S. Wolff, T. Woo, A. Yakovlev, T. Ziegler, ADF2014.01, SCM, Theoretical Chemistry, Vrije Universiteit, Amsterdam, The Netherlands http://www.scm.com 2014.

11. C. Adamo and V. Barone, J. Chem. Phys. 1999, 110, 6158.

12. E. van Lenthe and E. J. Baerends, J. Comput. Chem. 2003, 24, 1142.

13. ReSpect, version 4.0.0, 2016; Relativistic Spectroscopy DFT program of authors S. Komorovsky, M. Repisky, V. G. Malkin, O. L. Malkina, M. Kaupp, K. Ruud, with contributions from R. Bast, U. Ekstrom, M. Kadek, S. Knecht, L. Konecny, E. Malkin, I. Malkin-Ondik. (see www.respectprogram.org).

14. J. P. Perdew, K. Burke and M. Ernzerhof, Phys. Rev. Lett. 1996, 77, 3865.

15. K. G. Dyall, Unpublished Basis Sets, personal communication, 2016.

16. S. L. Hendershot, J. C. Jeffery, P. A. Jelliss, D. F. Mullica, E. L. Sappenfield, F. G. A. Stone, Inorg. Chem. 1996, 35, 6561. 